\title{
On the Magnetic Susceptibility of Fractal Magnetic Wires
}

\author{
S. O. Gladkov, S. B. Bogdanova* \\ Moscow Aviation Institute (State University of Aerospace Technologies), Volokolamskoe sh. 4, Moscow, 125993 Russia \\ *Corresponding Author: Sonjaf@list.ru
}

Copyright (C) 2013 Horizon Research Publishing All rights reserved.

\begin{abstract}
With the reasonable measure introduced the algorithm for computing the longitudinal component of the magnetic susceptibility of ferromagnetic fractal wire.
\end{abstract}

Keywords Fractal, Fractional Differentiation, Magnetic Susceptibility, Ferromagnetic.

\section{Introduction}

In describing physical properties of fractal objects always appear the problem of right dimension calculated physical characteristic's similar structures. This problem may be decided with helping valid introduction such notion as measure on fractal. For example, in deciding task about the calculation of longitudinal component of magnetic susceptibility tensor for ferromagnetic topologically one dimensionally fractal wire, in work [1] the accounting of measure was performed only phenomenological. The strong mathematic substantiation of measure in fractal we give in this work, and with it help of example sentence we describe the behavior longitudinal magnetic susceptibility of fractal wire, obviously illustrated the all receipt dependence by numerical calculation.

\section{Metrics and Measure of Fractal Object}

In describing physical properties of fractal structures, as was shown, for example, in [2,3], you must use the notion such as the fractional derivative. As for her introduction, the various authors introduce it differently, we also preferred it be introduced by the Fourier integral. When using the fractional derivative it will obviously violate the length dimension, so for example Gel'der suggested to introduce the length by a simple definition $\Delta y=\mu \Delta x^{\alpha}$, where $\mu-$ measure that provides the correct length dimensions $\Delta y$ and $\alpha-$ in the general case (not whole) the exponent.

In our case, the situation is somewhat more complicated, because it is a fractal, for which the concept of distance (metrics) between any two points in the case of topological dimension $d_{T}=1$ is somewhat unclear because of the vagueness of the distance scale selection.

However, as was shown in [4], we can introduce metrics on the fractal in the topologically one-dimensional case, which we are considering, according to the general definition

$$
d l^{2}=\mu^{2} g(x) d x^{2(1+\varepsilon)},
$$

where $\mu$-measure, and $g(x)$ - metric tensor, or more correctly, the only component of this tensor in the one-dimensional case.

From the condition of self-similarity of the distances between any two points on a fractal, considering that $x-$ the middle of this segment, you can enter a curved metric, assuming that

$$
g(x)=g^{\prime}\left(x^{\prime}\right)=g^{\prime}\left(\mu x^{\varepsilon}\right)=\mu^{2 \varepsilon} g^{\prime}(x)=\text { in } \operatorname{var},
$$

where $x^{\prime}=\mu x^{1+\varepsilon}$ is a nonlinear transformation.

On the other hand, from (1) with regard of (2) follows

$$
\begin{aligned}
& d l^{2}=\mu^{2} g(x) d x^{2(1+\varepsilon)}=\mu^{\prime 2} g^{\prime}\left(x^{\prime}\right) d x^{\prime 2(1+\varepsilon)} . \\
& =\mu^{\prime 2} \mu^{-2 \varepsilon} g(x) d x^{2(1+\varepsilon)}
\end{aligned}
$$

Hence $\mu^{\prime}=\mu^{1+\varepsilon}$. After we form the difference

$$
\mu^{\prime}-\mu=\mu\left(\left(l^{\varepsilon} \mu\right)^{\varepsilon}-1\right)
$$

and dividing it by $\varepsilon$, in the limit $\varepsilon \rightarrow 0$ we obtain the following renormalization-group equation

$$
\frac{d \mu}{d \varepsilon}=\mu \lim _{\varepsilon \rightarrow 0} \frac{\left(l^{\varepsilon} \mu\right)^{\varepsilon}-1}{\varepsilon}=\mu \ln \mu .
$$

Its solution is elementary and eventually we find

$$
\varepsilon=\int \frac{d \mu}{\mu \ln \mu}=\ln \ln \mu+\text { const }
$$

Hence

$$
\mu=e^{C e^{\varepsilon}},
$$

where $C$ - the constant of integration, which is separate for each fractal. Given the dimension this gives us the final expression for the measure

$$
\mu=\frac{e^{C e^{\varepsilon}}}{l^{\varepsilon}} .
$$


In case of any topologically one-dimensional lines (Koch curve, Sierpinski carpet, Peano curve, Menger sponge), $\varepsilon$ value can only be changed from zero to two. Therefore, further description of the physical properties of one-dimensional material fractal objects will be described using the dimensions

$$
d_{F}=1+\varepsilon,
$$

where, as noted above,

$$
0 \leq \varepsilon \leq 2 .
$$

Numerical analysis shows the validity of this assumption and gives us the right to impose a measure of the rule

$$
\mu=\frac{1}{l^{\varepsilon}} e^{C e^{\varepsilon}},
$$

where it is the finite length of the physical fractal $l$ is its feature distinguishing it from mathematics, the length of which, as you know, is infinite.

Due to the formula (9) we can describe now of the longitude magnetic susceptibility of the fractal objects.

\section{Magnetic Susceptibility of Finite Length Fractal.}

Proceeding from the general expression given in [1], we can assume the longitudinal component of the magnetic susceptibility of the ferromagnetic insulator to the form of

$$
\chi_{z z}(\omega)=\frac{\mu_{e}^{2}}{(2 \pi)^{3}} \frac{i \omega \bar{\tau}(\varepsilon)}{1-i \omega \bar{\tau}(\varepsilon)} \frac{\partial}{\partial T} \int \frac{k^{2} d k d \Omega}{e^{\frac{E_{\varepsilon}(k)}{T}}-1},
$$

where the magnon dispersion

$$
E_{\varepsilon}(k)=\alpha_{\varepsilon} M_{0}^{2}\left(k_{x}^{2+2 \varepsilon}+k_{y}^{2+2 \varepsilon}+k_{z}^{2+2 \varepsilon}\right)+\mu_{e}\left(H+H_{a}\right),
$$

and $d \Omega=\sin \theta d \theta d \varphi$ is an element of the corporal angle (about the parameter $\alpha_{\varepsilon}$ see below), the Boltzmann's constant $k_{B}=1$.

As to the expression (11) we'd like stop it more detail. The energy $E_{k}(\varepsilon)$ which appears in expression (11) must be calculated. To simplify the calculation we shell assume that the crystal has cubic structure. In this case we can use the phenomenological expression for Hamiltonian of magnetic ferromagnetic dielectric in the following appearance $[5,6]$

$$
H_{0}=\int_{V}\left[\frac{\alpha}{2}\left(\frac{\partial M_{i}}{\partial x_{k}}\right)^{2}-\vec{M} \vec{H}-\frac{\beta}{2} \vec{M}^{2}\right] d V \text {, }
$$

where $\alpha-$ is the exchange integral and $\beta-$ is a constant of the magnetic anisotropy.

The operation of usual differentiation for accordance coordinates of fractal objects in our case isn't inapplicable and we must use a new operation of fractal differentiation $A_{i}(i=x, y, z)$, which first was bring in the works $[7,8]$ due to the Fourier's integral

$$
\hat{A}_{x} f(\vec{r})=\int_{V} i k_{x}^{1+\varepsilon} f_{k} e^{i \vec{k} \vec{r}} d^{3} k
$$

Substituting of the partial derivatives in expression (12) on linear operators of fractal differentiation due to (13) we have

$$
\begin{array}{r}
\left(\frac{\partial M_{i}}{\partial x_{k}}\right)^{2} \Rightarrow\left(A_{i} \vec{M}\right)^{2}= \\
\frac{A M^{+} \cdot A M^{-}}{2}+\frac{A M^{-} \cdot A M^{-}}{2}+\left(A M_{z}\right)^{2} \cdot(14)
\end{array}
$$

Where

$$
M_{x}=\frac{M^{+}+M^{-}}{2}, M_{y}=\frac{M^{+}-M^{-}}{2 i} .
$$

An operators $M^{ \pm}$by the method of secondly quantization $[5,9]$ introduced. In the magnetic structures such operators of the Goldstein - Primakov's transformation determined:

$$
\begin{aligned}
& M^{+}=M_{0}\left(1-\frac{a^{+} a}{2 S}\right)^{\frac{1}{2}} a^{+}, \\
& M^{-}=M_{0} a\left(1-\frac{a^{+} a}{2 S}\right)^{\frac{1}{2}}, \\
& M_{z}=M_{0}\left(2 S-a^{+} a\right) .
\end{aligned}
$$

where $S_{\vec{k}}$ is a spin of magnetic atom of crystal lattice. In the $\vec{k}$ - space we have the following Fourier transformations

$$
\begin{gathered}
a^{+}(\vec{r})=\frac{1}{\sqrt{N}} \sum_{k} a_{k}^{+} e^{-i \vec{k} \vec{r}}, \\
a(\vec{r})=\frac{1}{\sqrt{N}} \sum_{k} a_{k} e^{i \vec{k} \vec{r}},
\end{gathered}
$$

where $N$ - is a number of lattices site.

Assuming that the value of atom spin is large $(S>>1)$ formulae (15) we can linear on the creation operator $a^{+}$ and annihilation operator $a$. In the result the Hamiltonian of our ferromagnetic system can be represent as

$$
H_{0}=\frac{1}{N} \sum_{\vec{k}} E_{k}(\varepsilon) a_{k}^{+} a_{k},
$$

where the energy of magnons in our fractal structure will

$$
\begin{aligned}
& E_{k}(\varepsilon)=\alpha_{\varepsilon} M_{0}^{2}\left(k_{x}^{2-2 \varepsilon}+k_{y}^{2-2 \varepsilon}+k_{z}^{2-2 \varepsilon}\right)+ \\
& +\mu_{e}\left(H_{0}+\beta M_{0}\right)
\end{aligned} .
$$


As we see from (19) the magnetic energy of fractal ferromagnetic is very anisotropic function of angular variable. The connect of the exchange constant $\alpha_{\varepsilon}$ with exchange integral are the following

$$
\alpha_{\varepsilon}=\mu^{2} a^{2} \frac{J_{e x}}{M_{0}^{2}}
$$

With goal to calculate of expression (10) we can introduce of the dimensionless value

$x=\frac{\mu^{2} a^{2} J_{e x} k^{2+2 \varepsilon} u(\theta, \varphi)+\mu_{e}\left(H+H_{a}\right)}{T}$, where $\mu=\frac{l^{\varepsilon}}{e^{C_{\varepsilon} e^{\varepsilon}}}-$ is measure of the fractal object, $l-$ its length obtained from the condition of "cutting" on the last iteration step break, the size of which is comparable to the magnon mean free path. Constant $C_{\varepsilon}$ is related to fractal dimension $\varepsilon$ and $\left.C_{\varepsilon}\right|_{\varepsilon=0}=0$, and the angle function

$$
u(\theta, \phi)=\sin ^{2+2 \varepsilon} \theta \cos ^{2+2 \varepsilon} \phi+\sin ^{2+2 \varepsilon} \theta \sin ^{2+2 \varepsilon} \phi+\cos ^{2+2 \varepsilon} \theta
$$

is obtained from (19) after transition to the spherical coordinate system. For the temperature range $\mu_{e}\left(H+H_{a}\right)<<T<<J_{e x}$ is taken to the following $\chi_{z z}(\omega)=\frac{3}{4 \pi^{2}} \frac{\mu_{e} M_{0}}{T(1+\varepsilon)}\left(\frac{T}{J_{e x}}\right)^{\frac{3}{2(1+\varepsilon)}} \frac{i \omega \bar{\tau}(\varepsilon)}{1-i \omega \bar{\tau}(\varepsilon)}\left(\frac{a^{\varepsilon}}{l}\right)^{\frac{3}{1+\varepsilon}} J_{1} J_{2}$

where $M_{0}=\frac{\mu_{e}}{a^{3}}-$ spontaneous magnetization of the ferromagnetic wire, the "easy axis" of which is directed along the wire axis. And the integral $J_{1}=\int_{\gamma}^{\infty} \frac{x^{\beta} d x}{e^{x}-1}$ with an exponent $\beta=\frac{1-2 \varepsilon}{2(1+\varepsilon)}$ and the lower limit of integration $\quad \gamma=\frac{\mu_{e}\left(H+H_{a}\right)}{T} \quad$ and integral $J_{2}=\frac{1}{4 \pi} \int_{0}^{2 \pi} \int_{0}^{\pi} \frac{\sin \theta d \theta d \varphi}{[u(\theta, \varphi)]^{\frac{3}{2(1+\varepsilon)}}}$.

As to the integral $J_{2}(\varepsilon)$ it value is complex and we can write that $J_{2}(\varepsilon)=f+i g$, where the functions $f(\varepsilon)$ and $g(\varepsilon)$ (after numerical integration) can illustrate by figure $1 \mathrm{a}$ and $1 \mathrm{~b}$, and table 1.

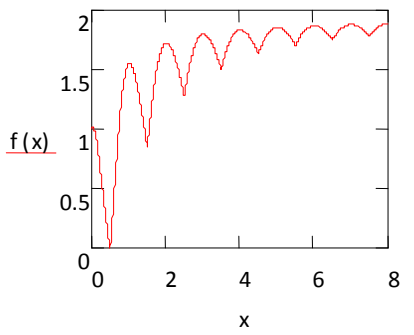

Figure. 1a

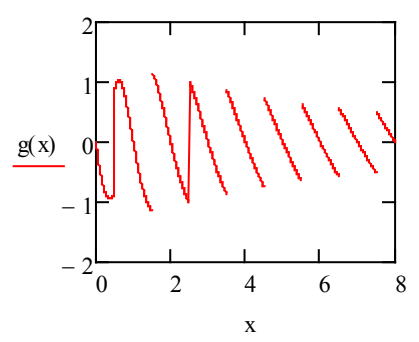

Figure $1 b$.

Table 1

\begin{tabular}{|c|c|c|c|c|}
\hline Curve & Koch & Sierpinski & Peano & Menger \\
\hline$\varepsilon$ & 0.26 & 0.89 & 1 & 1.73 \\
\hline$J_{2}$ & $0.57-86 \mathrm{i}$ & $1.4+0.41 \mathrm{i}$ & $1.5+0 \mathrm{i}$ & $1.42+0.64 \mathrm{i}$ \\
\hline$J_{1}$ & 3.16 & 7.41 & 8.45 & 15.01 \\
\hline
\end{tabular}

The integral $J_{1}$ was calculated in assumption, what the parameter $\gamma=\frac{\mu_{e}\left(H+H_{a}\right)}{T}=\frac{1+0.1}{100} \approx 0.01$ is small.

If separate in (21) of longitudinal component of an magnetic susceptibility tensor and imaginary part, we receive that $\chi_{z z}=\chi+i \psi$, where the functions

$$
\chi=\chi_{0}+\left(\chi_{0}-\chi_{\infty}\right) \frac{\omega \bar{\tau}}{1+\omega^{2} \bar{\tau}^{2}}\left|\omega \bar{\tau} g_{1}(\varepsilon)-g_{2}(\varepsilon)\right|
$$

$$
\psi=\left(\chi_{0}-\chi_{\infty}\right) \frac{\omega \bar{\tau}}{1+\omega^{2} \bar{\tau}^{2}}\left|\omega \bar{\tau} g_{2}(\varepsilon)+g_{1}(\varepsilon)\right|
$$

and the static magnetic susceptibility $\chi_{0}=\frac{3}{4 \pi^{2}} \frac{\mu_{e} M_{0}}{T(1+\varepsilon)}\left(\frac{T}{J_{e x}}\right)^{\frac{3}{2(1+\varepsilon)}} J_{1}$.

Indeed, the formulae (22) answer the question about quality dependence of magnetic susceptibility of fractal topologically one - dimensional ware as the function of dimension of the fractal object $\varepsilon$. The figure 2 show dependence of a real component $\chi_{z z}$ from $\varepsilon$, and the figure 3 corresponds dependence of imagine part $\chi_{z z}$. 


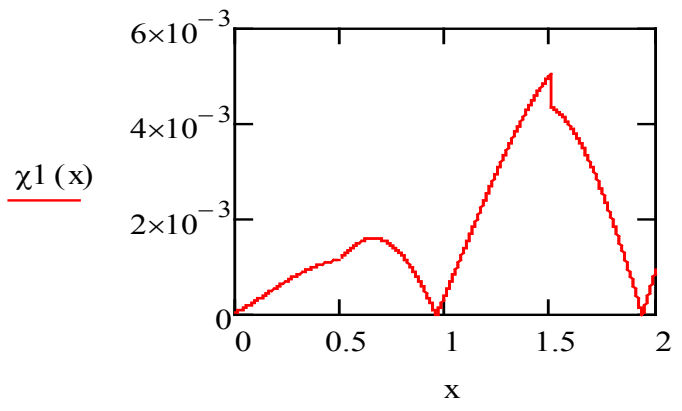

Figure 2.

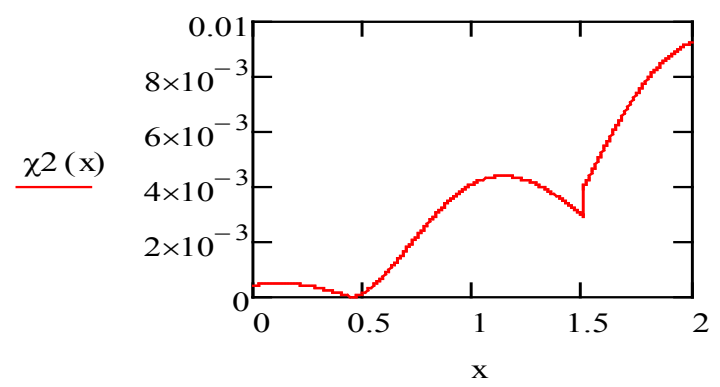

Figure 3.

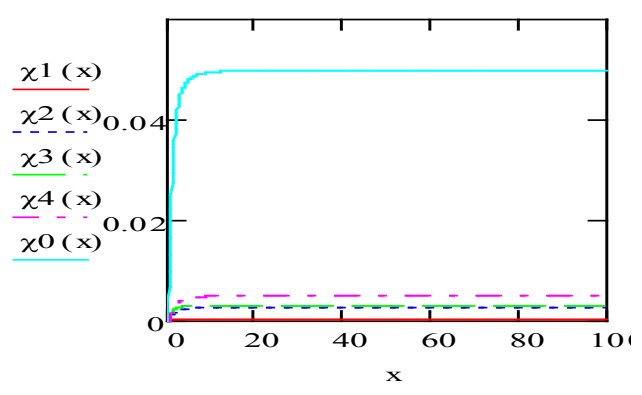

Figure 4.

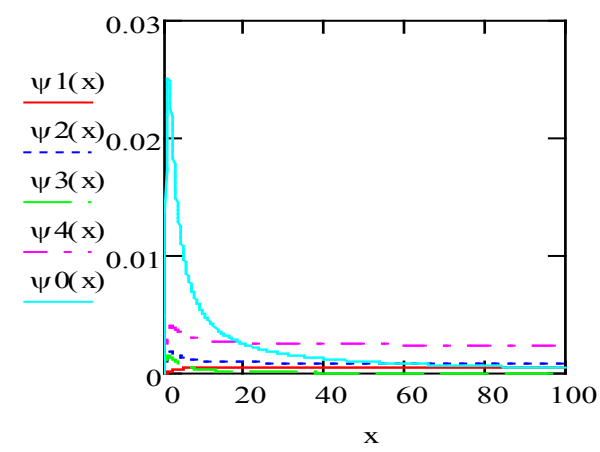

Figure 5.

So the formulae (21) allows us to evaluate the dissipative losses for any type of topologically one-dimensional physical fractal and will be very useful in the design and evaluation of the absorption of radio frequency waves by fractal antennas.

As we observe, in case if parameter $\varepsilon=0$ formulae (13) transfer to classic expression for longitudinal susceptibility [6]. Due to continues dependence $\chi_{z z}(\varepsilon)$, we can to compare these physical characteristics fractals and "smooth" curves on frequency and temperature. The picture 4 presents the behavior of frequencies dependences of the real part $\chi_{z z}$, and the picture 5 shows the behavior of the imagine part $\chi_{z z}$. We explane, that "smooth" curve correspond to the lines $\chi(0)$ and $\psi(0)$, Koch curve - $\chi(1)$ and $\psi(1)$, Sierpinski carpet - $\chi(2)$ and $\psi(2)$, Peano curve - $\chi(3)$ and $\psi(3)$, Menger sponge - $\chi(4)$ and $\psi(4)$. Where the parameter $x=\omega \bar{\tau}$.

\section{Conclusion}

In conclusion of our work we express the basic results of this research.

1. Due to the general principles of the theory renormalization group was received the strong substantiated analytical expression for measure.

2. It was calculated frequency and temperature dependence of longitudinal magnetic susceptibility for topologically one - dimensional fractal ware.

3. It was produced graphics results of numerical calculations of real and imagines components of the magnetic susceptibility.

\section{REFERENCES}

[1] Gladkov S.O., Bogdanova S.B. "On the theory of longitudinal magnetic susceptibility of quasi-three-dimensional ferromagnetic dielectrics". Physics State Solids. 2012, Vol.54. N 1. P.P.70 - 73.

[2] Gladkov S.O., Bogdanova S.B. On a generalization of kinetic equation for the case of non-integer dimension spaces. Condensed matter and interphases. 2011. V. 4. P.P. 423 426.

[3] Bogdanova S.B., Gladkov S.O. On the longitudinal magnetic susceptibility of fractal ferrodielectrics. Proceedings of the Academy of Sciences. Physical series. 2011. V.75. №10. P.P. $1418-1422$.

[4] Gladkov S.O. "Physics of composites: thermodynamic and dissipative properties.” M.: Nauka. 1999. 330 p.

[5] Ahiezer A.I., Bar'yahtar V.G., Peletminskii S.V. Spin Waves. Moscow, Science. 1967.

[6] Landau L.D., Lifshitz E.M. Course of Theoretical Physics, Vol. 8. Electrodynamics of Continuous Media. Moscow, Science. 1986.620 p.

[7] Gladkov S.O. To the theory of one-dimensional and quasi-one-dimensional heat conductivity. Journal of technical physics. 1997. V.67. №7. P.P. 8-12.

[8] Gladkov S.O. To the theory of hydrodynamic phenomena in quasi-one-dimensional systems. Journal of technical physics. 2001. V.71. №11. P.P. 130-132.

[9] Gladkov S.O. The kinetics of nuclear magnetically ordered systems. Physics Reports. 1989. V. 182. N 4, 5. P.P.211 - 364. 